\title{
Congenital Unilateral Absence of the Vas Deferens with Ipsilateral Renal Agenesis encountered during Laparoscopic Totally Extraperitoneal Inguinal Hernia Repair in an Adult Patient: A Case Report
}

\author{
Karel Smeyers ${ }^{1}$, Kor Hutting ${ }^{1}$, Ilse Hofmeester ${ }^{1}$, and Robert Prudhomme van Reine ${ }^{1}$ \\ ${ }^{1}$ Ziekenhuisgroep Twente
}

January 28, 2021

\begin{abstract}
We report the first incidental finding of congenital unilateral absence of the vas deferens and ipsilateral renal agenesis during endoscopic totally extraperitoneal inguinal hernia repair. The absence of the vasa deferentia occurs unilateral, associated with renal anomalies, or bilateral which has a genetic link to cystic fibrosis.
\end{abstract}

Congenital Unilateral Absence of the Vas Deferens with Ipsilateral Renal Agenesis encountered during Laparoscopic Totally Extraperitoneal Inguinal Hernia Repair in an Adult Patient: A Case Report

Karel M Smeyers, Kor H Hutting, Ilse Hofmeester, Robert Prudhomme van Reine

Department of surgery, Ziekenhuisgroep Twente, Almelo/Hengelo, the Netherlands

Mr. Karel Smeyers, M.D.

Department of surgery, Ziekenhuis groep Twente, Almelo/Hengelo

Zilvermeeuw 1, 7609 PP, Almelo, the Netherlands

Tel: +32494437930; e-mail: karel.smeyers@outlook.be

Congenital Unilateral Absence of the Vas Deferens with Ipsilateral Renal Agenesis encountered during Laparoscopic Totally Extraperitoneal Inguinal Hernia Repair in an Adult Patient: A Case Report

Abstract : We report the first incidental finding of congenital unilateral absence of the vas deferens and ipsilateral renal agenesis during endoscopic totally extraperitoneal inguinal hernia repair. The absence of the vasa deferentia occurs unilateral, associated with renal anomalies, or bilateral which has a genetic link to cystic fibrosis.

Key clinical message : Patients with unilateral absence of the vas deferens should be assessed for bilateral absence of the vasa deferentia by palpation in adults or laparoscopic exploration in pediatric patients. Ultrasonography should be performed to assess renal anomalies and genetic counselling is advised.

Keywords: Absence of vas deferens with renal agenesis; endoscopic hernia repair

Wordcount : 1774 (references excluded) 


\section{Introduction}

Inguinal hernia repairs are some of the most performed operations in general surgery worldwide. ${ }^{1} \mathrm{~A}$ rare and disconcerting finding during surgical exploration of the inguinal canal is absence of the vas deferens. This finding has been described in up to $1 \%$ of inguinal hernia repairs in pediatric patients ${ }^{2-6}$, but has not yet been described in inguinal hernia repairs in adult patients. We report the first incidental finding of unilateral absence of the vas deferens during elective laparoscopic totally extraperitoneal (TEP) inguinal hernia repair in an adult patient and describe the postoperative findings and management, clinical relevance, pathophysiology, and make recommendations for management based on literature and our experiences.

\section{Case report}

A 71-year-old man was referred to our surgical outpatient clinic because of bilateral symptomatic inguinal hernias. He had a relevant medical history of chronic obstructive pulmonary disease for over 20 years, a laparoscopic cholecystectomy because of symptomatic cholelithiasis 15 years earlier, idiopathic recurrent deep vein thrombosis in both legs and pulmonary embolism 10 months earlier, and right-sided epididymitis which was treated conservatively 5 months before. He had no known allergies and used Acenocoumarol (3 mg daily) and Salbutamol (200 mcg inhalations, 4 daily). Physical examination revealed left- and right-sided inguinal hernias which were easily reducible without symptoms of incarceration. His scrotum and testes were normal. Palpation of the vasa deferentia was not performed. We planned an elective laparoscopic TEP repair of both inguinal hernias and advised discontinuation of the Acenocoumarol 24 hours before surgery with bridging therapy (Dalteparin 25,000 IE/mL, $0.3 \mathrm{~mL}$ twice daily).

We performed a laparoscopic TEP repair of both inguinal hernias, which were found to be direct types, under general anaesthesia using a conventional three-port technique. Intra-operatively we observed left-sided absence of the vas deferens. Normal inferior epigastric vessels and testicular vessels were found bilaterally. Iatrogenic damage was not observed nor suspected. The procedure and post-operative recovery were uneventful.

Postoperative ultrasonography revealed a normal bladder, prostate, and right-sided kidney and ureter, but absence of the left-sided kidney and ureter. A nephrologist was consulted, but since the patient was asymptomatic, normotensive, and had normal levels of serum creatinine and urinary protein, no further management was advised besides annual surveillance and recommendations regarding cardiovascular disease prevention.

\section{Discussion}

Congenital unilateral absence of the vas deferens (CUAVD) is mostly diagnosed incidentally since it is rarely symptomatic nor a cause of infertility. ${ }^{7}$ Based on vasectomy data, the incidence in the general population is estimated to be $0.5 \%-1.0 \% .2$ CUAVD is found on the left side in $66 \%{ }^{7}$ The incidental finding of CUAVD during inguinal hernia repair is clinically relevant for the operating surgeon, firstly because it is associated with renal abnormalities. Ipsilateral renal agenesis is found in $74 \%-79 \%$ of men with CUAVD and in one third of these men abnormalities of the contralateral solitary kidney are also present. ${ }^{7}{ }^{8}$ Secondly, when absence of the vas deferens is found during unilateral surgical exploration of the inguinal canal, the contralateral vas deferens could be absent as well as a part of congenital bilateral absence of the vasa deferentia (CBAVD).

CBAVD naturally leads to azoospermia and is therefore mostly diagnosed during infertility examination. It is estimated to be the cause of azoospermia in $1 \%-6 \%$ of infertile men. ${ }^{7}$ Renal anomalies occur with CBAVD but are found less often (10-21\%) than in men with CUAVD. ${ }^{9,10}$ CBAVD is genetically linked with cystic fibrosis $(\mathrm{CF})$ and is almost invariably present in male CF patients. ${ }^{11-14}$ Male CF patients rarely have 
renal anomalies. ${ }^{5,9,15}$ Men with CBAVD without renal anomalies or clinical signs of CF are considered to have a mild, genital form of $\mathrm{CF}$ since $\mathrm{CF}$ transmembrane conductance regulator (CFTR) gene mutations have been found in $64 \%-75 \%$ of these men. ${ }^{7,10,11,16-18}$ CTFR gene mutations have rarely been described in men with CBAVD and renal anomalies and have never been described in fertile men with CUAVD and renal anomalies but have been described in men with CUAVD and associated infertility due to non-patency of the solitary vas deferens. ${ }^{19-21}$

The co-occurrence of CUAVD and CBAVD with renal anomalies originates from the close association of the reproductive and urinary tracts during embryogenesis. Between the fourth and sixth week of gestation, the mesonephric (Wolffian) ducts connect the mesonephros to the urogenital sinus and form ureteric buds, which later differentiate into the ureter, renal pelvis and metanephric kidneys. After seven weeks of gestation, the mesonephric ducts separate from the differentiating ureteric buds. The proximal parts of the mesonephric ducts remain fixed to the urogenital sinus to form the ejaculatory ducts, seminal vesicles and juxta-urethral vasa deferentia whilst the upper parts form the corpus and cauda epididymis and the scrotal and inguinal vasa deferentia. ${ }^{2,8,22}$ Because of this separation of the primitive reproductive and urinary tracts after 7 weeks of gestation, CUAVD or CBAVD with renal anomalies is caused by a genetic or toxic disturbance in the development of the mesonephric ductal system before the seventh week of gestation, whilst CUAVD or CBAVD without renal anomalies is caused by a later developmental disturbance. The caput epididymis, vasa efferentia and testes develop separately from the mesonephros and are therefore never affected. ${ }^{2,8,22}$ It remains unclear how renal agenesis occurs unilaterally in CBAVD, and why unilateral renal agenesis does not occur in CBAVD in CF patients. The exact pathophysiology of CBAVD and CUAVD, with or without renal anomalies, thus remains poorly understood.

The reported patient had children of his own and never experienced symptoms suggestive of CUAVD. His medical history stated epididymitis 5 months before the operation, but this was right-sided and was therefore considered coincidental. Palpation of the vasa deferentia was omitted in the pre-operative physical examination. In retrospect, this could have revealed CUAVD and associated ipsilateral renal agenesis preoperatively. Even though the reported patient was asymptomatic and had a normal renal function, preoperative knowledge of CUAVD and potential unilateral renal agenesis is important to prevent problems in patients with a decreased renal function and to urge the surgeon to take even more care to prevent iatrogenic damage to the contralateral solitary vas deferens. Some studies suggest that a congenital solitary kidney increases the risks of hypertension, proteinuria and mild renal insufficiency ${ }^{23}$ and therefore we advised the patient long-term annual surveillance of blood pressure, urinary protein and serum creatinine. Fortunately, patient survival has never been found to be less in patients with a congenital solitary kidney. ${ }^{23}$ The reported patient had no history or clinical signs of CF. His family history revealed no CF, infertility or abnormalities of the urinary or reproductive tracts. No genetic investigations regarding CFTR gene mutations were required in the reported patient since laparoscopic evaluation of the contralateral inguinal canal revealed an anatomically normal vas deferens, ruling out CBAVD, with normal fertility and ipsilateral renal agenesis.

Based on the literature and our experiences in the reported case we state recommendations for surgeons who encounter CUAVD or CBAVD incidentally during inguinal hernia repairs. Firstly, we advise thorough pre-operative physical examination before inguinal hernia repair in male patients, including palpation of the vasa deferentia to assess CUAVD or CBAVD. Vasa deferentia are easily palpable in adult patients and therefore CUAVD or CBAVD can be diagnosed by physical examination without imaging or surgical exploration. ${ }^{8}$ Therefore, when absence of the vas deferens is found during unilateral inguinal hernia repair and pre-operative bilateral palpation of the vasa deferentia has been omitted, we recommend palpation of the contralateral vas deferens to assess for CBAVD. In our opinion, surgical exploration for assessment of the contralateral vas deferens is not indicated when CUAVD is found in adult patients, since this could increase morbidity and the risk of infertility due to iatrogenic damage. For pediatric patients, however, some authors advise concomitant laparoscopic evaluation of the contralateral vas deferens when unilateral absence of the vas deferens is found.[5] Naturally, adequate palpation of the vasa deferentia is more difficult in pediatric patients whilst accurate diagnosis of CBAVD is important because of the resulting infertility and potential presence of $\mathrm{CF} .{ }^{5}$ We therefore agree with recommending surgical exploration of the contralateral inguinal canal to 
assess CBAVD in pediatric patients, preferably by laparoscopy in the same general anaesthetic. Naturally, the surgeon should explain the possibility of required contralateral exploration of the inguinal canal to the parents and pediatric patient pre-operatively. When this has been omitted, one could offer contralateral exploration of the inguinal canal either intra-operatively to the parents by telephone or postoperatively to be planned as a second laparoscopy. Secondly, we recommend postoperative renal ultrasonography in all men with CUAVD or CBAVD to assess unilateral renal agenesis, renal abnormalities or renal ectopia. When renal anomalies are found we advise to consult a nephrologist for management or long-term surveillance of blood pressure, urinary protein and serum creatinine. Thirdly, we recommend genetic evaluation of CFTR gene mutations in when CBAVD is found in either pediatric or adult patients, or when CUAVD is found in infertile men. Finally, we advise adequate counselling when CBAVD is found in pediatric patients regarding infertility and further genetic evaluation with potentially disconcerting outcomes for relatives.

In conclusion, we report the first incidental finding of CUAVD with ipsilateral renal agenesis during laparoscopic TEP repair of bilateral inguinal hernias in an adult patient. The finding of unilateral absence of the vas deferens is clinically relevant because it could either indicate CUAVD, in which associated renal anomalies are found in a majority of men, or CBAVD, in which associated renal anomalies are found in a minority of men but which leads to azoospermia and often has a genetic link to CF. Adequate physical examination is important to assess for CUAVD or CBAVD preoperatively. When absence of the vas deferens is found during unilateral laparoscopic inguinal hernia repair in an adult patient we advise palpation of the contralateral vas deferens to assess the contralateral vas deferens and to rule out CBAVD. When absence of the vas deferens is found during unilateral laparoscopic inguinal hernia repair in pediatric patients, concomitant laparoscopic evaluation of the contralateral inguinal canal is advisable. We advise renal ultrasonography in all patients with CUAVD or CBAVD and to consult a nephrologist when renal anomalies are present. Genetic evaluation of CFTR gene mutations is advisable in all children or men with CBAVD or in men with CUAVD who are infertile.

\section{Acknowledgements}

None

\section{Disclosure Statement}

No competing financial interests exist.

\section{Author Contributions}

Karel Smeyers: Clinical follow up of patient presented in case report; writing and submitting the case report.

Kor Hutting: Clinical admittance of patient presented in case report; writing the case report.

Ilse Hofmeester: Assisting during operative procedure of patient presented; advise in writing case report

Robert Prudhomme van Reine: Surgeon performing procedure of patient presented in case; supervising writing of case report.

\section{References:}

1. Devlin HB: Trends in hernia surgery in the land of Astley Cooper. In: Soper NJ, Ed. Problems in General Surgery. Philadephia: Lippincott-Raven, 1995, pp. 85-92.

2. Lukash F, Zwiren GT, Andrews HG. Significance of absent vas deferens at hernia repair in infants and children. J Pediatr Surg 1975;10:765-9. 
3. Shepherd G, Rajimwale A. Embryology of the Absent Vas Supported by 2 Cases of Congenital Unilateral Absence of Vas With Varied Associations. Urol case reports 2014;2:49-50.

4. Martin RA, Jones KL, Downey EC. Congenital absence of the vas deferens: recurrence in a family. Am J Med Genet 1992;42:714-5.

5. Lane VA, Scammell S, West N, et al. Congenital absence of the vas deferens and unilateral renal agenesis: implications for patient and family. Pediatr Surg Int 2014;30:733-6.

6. Abdelmohsen SM, Osman MA. Congenital vanished distal part of the right vas deferens, a case report. Urol case reports 2017;15:46-7.

7. Weiske WH, Salzler N, Schroeder-Printzen I, et al. Clinical findings in congenital absence of the vasa deferentia. Andrologia 2000;32:13-8.

8. Donohue RE, Fauver HE. Unilateral absence of the vas deferens. A useful clinical sign. JAMA 1989;261:1180-2.

9. McCallum TJ, Milunsky JM, Munarriz R, et al. Unilateral renal agenesis associated with congenital bilateral absence of the vas deferens: phenotypic findings and genetic considerations. Hum Reprod. $2001 ; 16: 282-8$.

10. Augarten A, Yahav Y, Kerem BS, et al. Congenital bilateral absence of vas deferens in the absence of cystic fibrosis. Lancet 1994;344:1473-4.

11. Dörk T, Dworniczak B, Aulehla-Scholz C, et al. Distinct spectrum of CFTR gene mutations in congenital absence of vas deferens. Hum Genet 1997;100:365-377.

12. Anguiano A, Oates RD, Amos JA, et al. Congenital bilateral absence of the vas deferens. A primarily genital form of cystic fibrosis. JAMA 1992;267:1794-7.

13. Kaplan E, Shwachman H, Perlmutter AD, et al. Reproductive failure in males with cystic fibrosis. N Engl J Med 1968;279:65-9.

14. Holsclaw DS, Perlmutter AD, Jockin H, et al. Genital abnormalities in male patients with cystic fibrosis. J Urol 1971;106:568-574.

15. Gaillard DA, Carré-Pigeon F, Lallemand A. Normal vas deferens in fetuses with cystic fibrosis. J Urol 1997;158:1549-1552.

16. Oates RD, Amos JA. The genetic basis of congenital bilateral absence of the vas deferens and cystic fibrosis. J Androl 1994;15:1-8.

17. De Braekeleer M, Férec C. Mutations in the cystic fibrosis gene in men with congenital bilateral absence of the vas deferens. Mol Hum Reprod 1996;2:669-677.

18. Schwarzer JU, Schwarz M. Significance of CFTR gene mutations in patients with congenital aplasia of vas deferens with special regard to renal aplasia. Andrologia 2012;44:305-7.

19. Daudin M, Bieth E, Bujan L, et al. Congenital bilateral absence of the vas deferens: clinical characteristics, biological parameters, cystic fibrosis transmembrane conductance regulator gene mutations, and implications for genetic counseling. Fertil Steril 2000;74:1164-1174.

20. Mickle J, Milunsky A, Amos JA, et al. Congenital unilateral absence of the vas deferens: a heterogeneous disorder with two distinct subpopulations based upon aetiology and mutational status of the cystic fibrosis gene. Hum Reprod 1995;10:1728-1735.

21. Kolettis PN. The evaluation and management of the azoospermic patient. J Androl 2002;23:293-305.

22. Deane AM, May RE. Absent vas deferens in association with renal abnormalities. Br J Urol 1982;54:298-9. 
23. Shapiro E, Goldfarb DA, Ritchey ML. The congenital and acquired solitary kidney. Rev Urol 2003;5:2-8. 